\title{
Effect of coexisting diabetes mellitus and chronic kidney disease on mortality of cirrhotic patients with esophageal variceal bleeding
}

\author{
Chia-Chi Lung ${ }^{1,2^{*}}$, Zhi-Hong Jian ${ }^{1}$, Jing-Yang Huang ${ }^{1}$ and Oswald Ndi Nfor ${ }^{1}$
}

\begin{abstract}
Background: Esophageal variceal bleeding (EVB) is a serious and common complication of cirrhosis. Diabetes mellitus (DM) and chronic kidney disease (CKD) increase mortality in patients with cirrhosis. However, whether coexisting DM and CKD increase mortality in cirrhotic patients with EVB remains unclear.

Methods: We enrolled cirrhotic patients hospitalized with the first presentation of EVB from 2005 through 2010 using Longitudinal Health Insurance Database 2005. The hazard ratios (HRs) of 42-day and one-year EVB mortality were calculated using Cox regression model.

Results: We identified 888 patients hospitalized with the first presentation of EVB. Among the cirrhotic patients with EVB, all-cause mortality at 42-day and one-year were 21.3 and $45.0 \%$, respectively. The respective HRs for the 42-day and one-year mortality were 1.80 (95\% confidence interval [Cl], 1.10-2.97) and 1.52 (95 \% Cl, 1.06-2.17) for patients with CKD and $0.79(95 \% \mathrm{Cl}, 0.57-1.10)$ and 0.88 (95\% Cl, 0.71-1.09) for patients with DM. Specifically, coexisting CKD and DM increased the 42-day and one-year mortality with respective HRs of $1.99(95 \% \mathrm{Cl}, 1.03-3.84)$ and $1.84(95 \% \mathrm{Cl}, 1.14-2.98)$ compared with those without CKD and DM. The HRs for 42-day and 1-year mortality in female patients with DM and CKD were $4.03(95 \% \mathrm{Cl}, 1.40-11.59)$ and $2.84(95 \% \mathrm{Cl}, 1.31-6.14)$ respectively, and were $2.93(95 \% \mathrm{Cl}, 1.14-7.57)$ and $2.42(95 \% \mathrm{Cl}, 1.28-4.57)$ in male patients with DM and CKD.
\end{abstract}

Conclusion: We identified that coexisting DM and CKD increased risk of mortality at 42 days and 1 year following EVB.

Keywords: Chronic kidney disease, Cirrhosis, Diabetes mellitus, Esophageal variceal bleeding

\section{Background}

Bleeding from esophageal varices is a life-threatening condition with an annual mortality of $57 \%$. Nearly half these deaths occur within 6 weeks from the initial episode of bleeding [1]. Various factors have been proposed as predictors of outcome of variceal bleeding, some of which include age, gender, stage of cirrhosis, etiology, and associated conditions like renal failure and diabetes mellitus (DM) [2-5].

\footnotetext{
* Correspondence: dinoljc@csmu.edu.tw

${ }^{1}$ Department of Public Health and Institute of Public Health, Chung Shan Medical University, Taichung City, Taiwan

${ }^{2}$ Department of Family and Community Medicine, Chung Shan Medical

University Hospital, Taichung City, Taiwan
}

Renal function is a critical prognostic factor in cirrhotic patients with esophageal variceal bleeding (EVB) $[6,7]$. Patients with chronic kidney disease (CKD) have many long-term complications, such as increased immunocompromised status [8], as well as an increased risk of metabolic disorders [9], and cardiovascular events [10]. Physiological mechanisms contributing to an increased bleeding tendency included uremic platelet dysfunction, use of antiplatelet agents, and anticoagulants [11, 12]. In addition, usage of aspirin is associated with the occurrence of EVB in cirrhotic patients [13].

The Verona Diabetes Study, a population-based study with 7,148 patients with DM, showed an increased risk of mortality from chronic liver disease and cirrhosis compared with the general population [14]. Insulin resistance, 
a characteristic feature of DM, has been proven to be a predictor of portal hypertension [15] and the development of esophageal varices [16]. In a hospital-based study with 146 patients with cirrhosis, DM significantly correlated with gastroesophageal variceal bleeding [2].

The presence of metabolic syndrome and the number of metabolic syndrome components have been associated with higher prevalence of CKD [9]. CKD is one of the most common long-term complications of DM [17]. The prevalence of DM and CKD in Taiwan has been reported as 7,570 and 892 per 100,000 population, respectively $[18,19]$. How DM and/or CKD per se, is/are independent mortality risk factors, and how they further increase the risk of mortality is still unclear. This study aimed to investigate DM and CKD on mortality of cirrhotic patients with first presentation of EVB.

\section{Methods}

\section{Data source}

This retrospective cohort study used data from the Longitudinal Health Insurance Database 2005 (LHID2005), which is a subset of the National Health Insurance Research Database (NHIRD). The LHID2005 database was derived by the Bureau of National Health Insurance, Ministry of Health and Welfare of Taiwan and maintained by the National Health Research Institutes so as to make it accessible for research purposes. The LHID2005 is broadly used in academic studies [20-23]. LHID2005 contains all the original claims data of one million out of 23 million National Health Insurance enrollees, randomly sampled from the year 2005 registry for beneficiaries of the NHIRD. There was no significant difference in the age and sex distribution between patients in the LHID2005 and the original NHIRD [24]. The use of the data was reviewed and granted by the National Health Research Institutes. The source data was encrypted and the data extracted was anonymous. This study was approved by the Institutional Review Board of the Chung-Shan Medical University Hospital, Taiwan.

\section{Patient identification}

This retrospective study included cirrhotic patients who were hospitalized with a first presentation of EVB between 2005 and 2010. Subjects with incomplete information, such as sex and registry data were excluded. EVB was confirmed by the International Classification of Diseases, Ninth Revision, Clinical Modification (ICD-9-CM) code (ICD-9-CM code 456.0 and 456.20) and esophageal variceal ligation or sclerotherapy.

\section{Variables of exposure}

To reduce bias, the diagnoses of comorbidities were confirmed by more than two outpatient visits or one admission in 1 year. Comorbidities were defined using the following ICD-9-CM codes: DM (250), CKD (585 and $586)$, hepatitis B virus (HBV) infection (070.2, 070.3, and V02.61), hepatitis $\mathrm{C}$ virus (HCV) infection (070.41, 070.44, 070.51, 070.54, 070.7, and V02.62), alcohol-related disorders (291, 303, 305.00-305.03, and 571.0-571.3), hepatocellular carcinoma (HCC) (155.0 and 155.2), ascites (789.5 or ICD-9 Volume 3 procedure code 54.91), hepatic encephalopathy (572.2), spontaneous bacterial peritonitis (SBP) $(567.2,567.8$, or 567.9 , excluding the procedure codes for the abdominal surgery), chronic obstructive pulmonary disease (COPD) (490, 491, 492, 494, and 496), asthma (493), pulmonary tuberculosis (TB) (010 - 012), acute coronary syndrome (410-414)), cerebrovascular accident (430-438), and bacterial infections. The bacterial infections during hospitalization included pneumonia (ICD-9-CM 481-487, excluding 484), liver abscess (ICD-9-CM 572.0), empyema (ICD-9-CM 510), cellulitis (ICD-9-CM 681 and 682), necrotizing fasciitis (ICD-9-CM 728.86), central nervous system infection (ICD-9-CM 324 and 320), sepsis (ICD-9-CM 038 and 790.7), infective endocarditis (ICD-9-CM 421), urinary tract infection (ICD-9-CM 590.1, 595.0, 595.9, and 599.0), biliary tract infection (ICD-9-CM 574.00, 574.01, 574.30, 574.31, 574.60, 574.61, 574.80, 574.81, 575.0, and 576.1), septic arthritis (ICD-9-CM 711), and perianal abscess (ICD-9-CM 566).

\section{Statistical analysis}

All analyses were made using SAS 9.3 software (SAS Institute, Cary, $\mathrm{NC}$ ). Chi square test was used to exam the differences in sociodemographic characteristics and comorbidities. Multivariate Cox proportional hazards regression was performed to determine mortality for independent variables, such as sex, age, low income, urbanization, comorbidities, etiology, and complications of cirrhosis. Furthermore, in order to evaluate the effect of coexisting DM and CKD on all-cause mortality, 4 separate models were conducted: 42-day mortality of all patients (Model 1), 42-day mortality stratified by gender (Model 2), 1-year mortality of all patients (Model 3), and 1-year mortality stratified by gender (Model 4). All comparisons with a $p$-value $<0.05$ were considered statistically significant.

\section{Results}

The demographic characteristics and comorbidities of cirrhotic patients with EVB are listed in Table 1. The 42-day and 1-year EVB mortalities were 21.3 and $45.0 \%$, respectively. HBV, CKD, HCC, and hospitalization due to ascites, hepatic encephalopathy, and SBP were more common in non-survivors. The possible etiologies of CKD were as follows: diabetes alone, 3 cases (4.6\%), diabetes + hypertension, $8(12.3 \%)$, diabetes + hypertension + coronary artery disease, 2 (3.1\%), diabetes + hypertension + hyperlipidemia, 2 (3.1\%), diabetes + hypertension + 
Table 1 Characteristics of cirrhotic patients with first esophageal variceal bleeding and mortality, Taiwan, 2005-2010

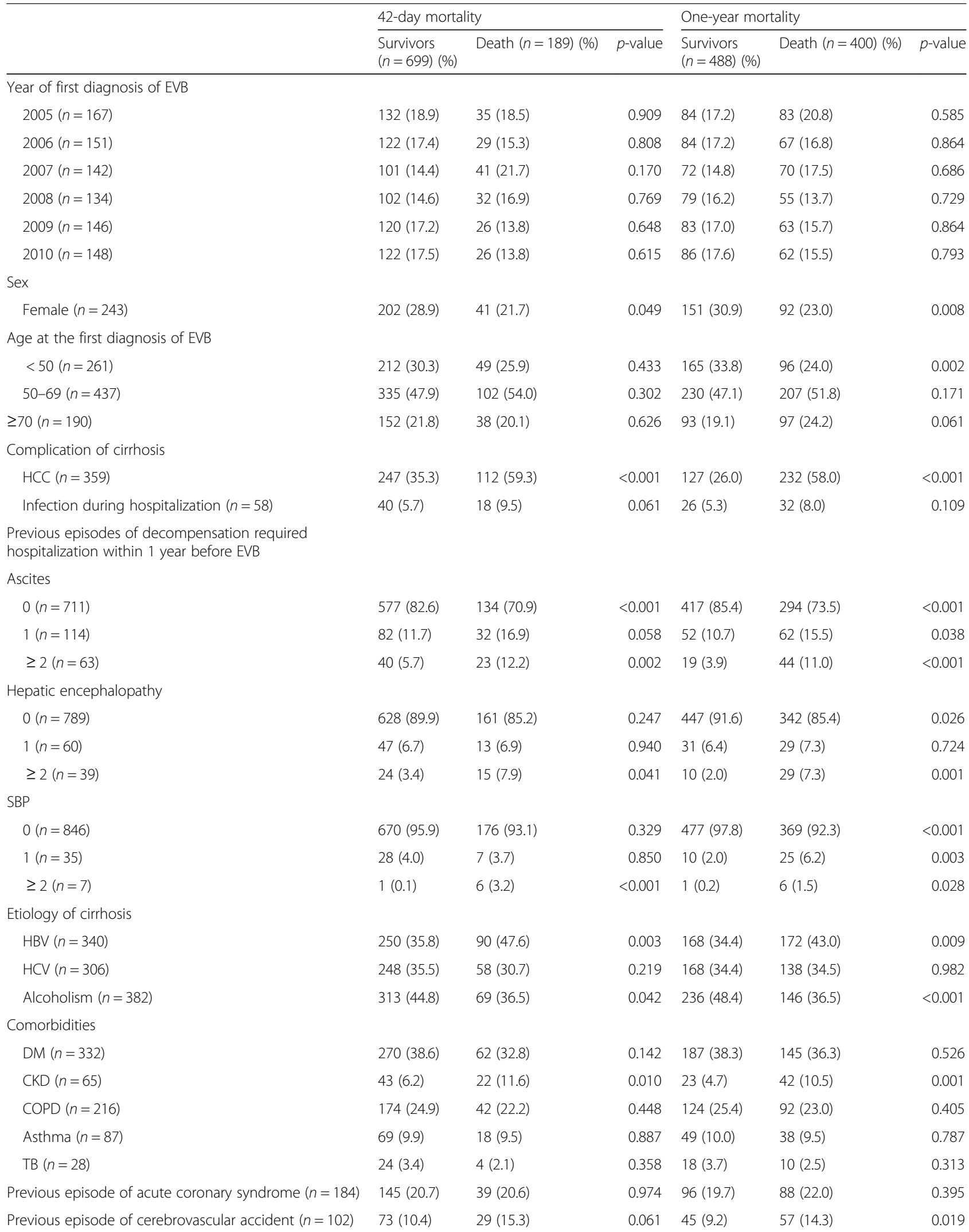


Table 1 Characteristics of cirrhotic patients with first esophageal variceal bleeding and mortality, Taiwan, 2005 -2010 (Continued)

\begin{tabular}{|c|c|c|c|c|c|c|}
\hline \multicolumn{7}{|l|}{ Low income } \\
\hline Yes $(n=24)$ & $20(2.9)$ & $4(2.1)$ & 0.575 & $7(1.4)$ & $17(4.3)$ & 0.010 \\
\hline \multicolumn{7}{|l|}{ Urbanization } \\
\hline Urban $(n=438)$ & 339 (48.5) & 99 (52.4) & 0.357 & $246(50.4)$ & 192 (48.0) & 0.475 \\
\hline Normal $(n=316)$ & $243(34.8)$ & 73 (38.6) & 0.357 & 160 (32.8) & 156 (39.0) & 0.120 \\
\hline Rural $(n=134)$ & $117(16.7)$ & $17(9.0)$ & 0.030 & $82(16.8)$ & $52(13.0)$ & 0.214 \\
\hline
\end{tabular}

CKD chronic kidney disease, COPD chronic obstructive pulmonary disease, DM diabetes mellitus, EVB esophageal variceal bleeding, $H B V$ hepatitis $B$ virus, $H C C$ hepatocellular carcinoma, HCV hepatitis C virus, SBP spontaneous bacterial peritonitis, TB pulmonary tuberculosis

coronary artery disease + hyperlipidemia, 5 (7.7 \%), diabetes + other etiologies, 4 (6.1\%), and etiologies other than diabetes, 41 (63.1\%). For CKD patients with or without DM, the 42-day mortalities were $41.7 \%$ $(10 / 24)$ and $29.3 \%(12 / 41)$, and the 1-year mortality were $79.2 \%(19 / 24)$ and $56.1 \%(23 / 41)$, respectively (data not shown).

Table 2 shows the HRs for 42-day and 1-year mortality. At 42 days following EVB, the risk of mortality was high in patients with CKD (hazard ratio [HR], 1.80; $95 \%$ confidence interval [CI], 1.10-2.97], HCC (HR, 2.13; $95 \%$ CI, 1.54-2.95), and previous hospitalization due to ascites and SBP. Similarly, at 1 year following EVB, risk of mortality was also high in men (HR,1.54; $95 \% \mathrm{CI}$, 1.19-2.00), CKD (HR,1.52; $95 \% \mathrm{CI}, 1.06-2.17)$, HCC (HR, 2.48; 95\%CI, 1.99-3.10), infections during hospitalization (HR:1.50; 95 \% CI, 1.03-2.18), and previous hospitalization due to ascites and SBP.

Table 3 illustrates the adjusted HRs of EVB mortality at 42 days and 1 year in patients with either DM, CKD, or both by gender. For 42-day mortality (Model 1), the HRs were 1.99 (95\% CI, 1.03-3.84) and 0.72 (95\%CI, 0.50-1.02) among DM patients with or without CKD, respectively. The HR for patients with CKD was 1.25 (95\% CI, 0.62-2.52). When stratified by gender and disease combinations (Model 2), the HRs were higher in female diabetic patients with CKD (HR, 4.03; 95 \% CI, 1.40-11.59) and male diabetic patients with CKD (HR, 2.93; 95 \% CI, 1.14-7.57).

For 1-year mortality, the HRs were 1.84 (95\% CI, 1.14-2.98) and 0.80 (95\% CI, 0.64-1.02) in diabetic patients with or without CKD (Model 3). The HR for patients with CKD was 1.07 (95\% CI, 0.65-1.76). There was significant interaction between DM and CKD ( $p=$ 0.028). In Model 4, the HRs for male and female diabetic patients with CKD were 2.84 (95\% CI, 1.31-6.14) and 2.42 (95\% CI, 1.28-4.57), respectively.

\section{Discussion}

We found that coexistence of CKD and DM was independently associated with 42-day and 1-year mortality in both sexes. These risk factors are easy to identify following the initial EVB event and are valuable for predicting clinical outcomes. They may also be useful for guiding the clinical management of cirrhotic patients with EVB. Identifying patients at high risk will be important for cost-effective management of EVB.

Globally, $57 \%$ of cirrhosis is attributable to either hepatitis B (30\%) or hepatitis C (27\%) [25]. Alcohol consumption is another important cause, accounting for about $20 \%$ of the cases. The seroprevalence of HBV and HCV was 17.3 and $4.4 \%$ in Taiwan, respectively [26]. More than $70 \%$ of cirrhosis and HCC were the sequelae of chronic HBV infection in 1990s [27]. Our results showed that HBV infection was higher in non-survivors and the prevalence of HBV infection in HCC was $46.2 \%$. When HBV infection was put into the multivariate model for analyses, there was no significant association between HBV infection and EVB mortality. The efficacy of universal immunization has been proven with substantial reductions of HBV carriage in children, adolescents and young adults since 1984 [28]. HCC in Taiwan also falls after universal hepatitis B vaccination [29]. We also showed improvement in the 42-day mortality rate over time perhaps due to treatment advances, such as variceal ligation, appropriate vasoconstrictor usage, and antiviral treatment for the underlying cirrhosis [30, 31].

Bleeding from ruptured esophageal varices is the most severe complication of cirrhosis and 6-week mortality rates have been reported to be $15-20 \%$ [30]. The inpatient bleeding rate among cirrhotic patients has been reported as $13 \%$ [32]. Around 30 to $60 \%$ of cirrhotic patients suffer from DM with insulin resistance and hyperinsulinemia [33]. The presence of DM appears to be associated with failure to control esophageal variceal bleeding and re-bleeding [34]. Hyperglycemia induces splanchnic hyperemia, increases portal pressure and azygos vein blood flow, and may increase the risk of variceal bleeding $[35,36]$. However, in this study, DM individually failed to show significant association with 42-day and 1 -year mortality.

In an analysis involving 2,592 cirrhotic patients hospitalized with SBP in 2004, the respective HRs for 30-day and 1-year mortality were 1.37 (95\%CI, 0.85-2.21) and 1.37 (95\%CI, 1.01-1.84) in patients with CKD [37]. Increased long-term mortality rates of SBP in cirrhotic 
Table 2 Estimation of hazard ratios of mortality in patients with esophageal variceal bleeding between 2005 and 2010 using cox proportional model

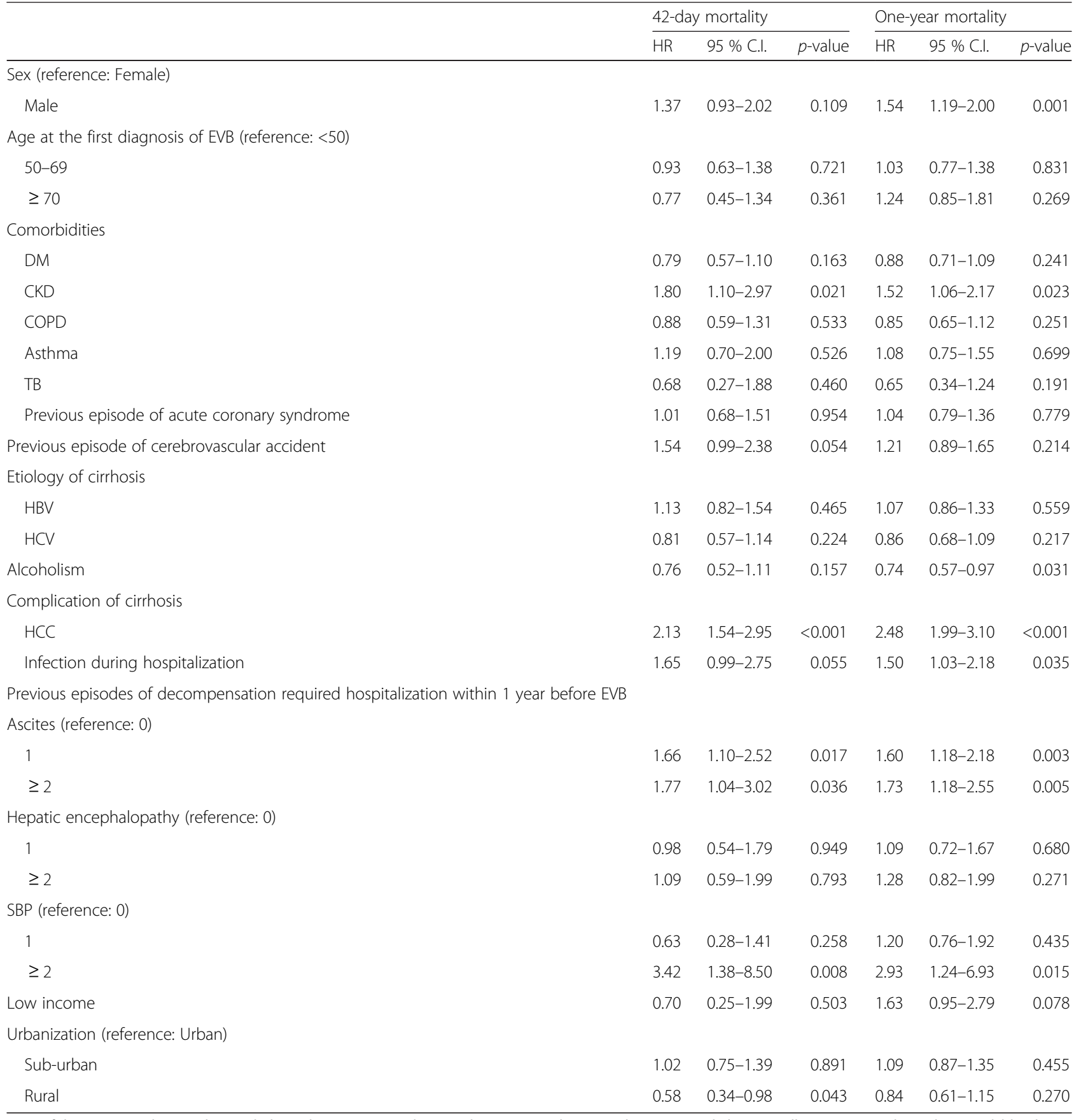

$C I$ confidence interval, $C K D$ chronic kidney disease, COPD chronic obstructive pulmonary disease, DM diabetes mellitus, EVB esophageal variceal bleeding, $H B V$ hepatitis B virus, HCC hepatocellular carcinoma, HCV hepatitis C virus, HR hazard ratio, SBP spontaneous bacterial peritonitis, TB pulmonary tuberculosis

patients may be attributed to the impaired immune functions caused by CKD [38]. Hung et al. evaluated 4,932 cirrhotic patients with hepatic encephalopathy and showed that the adjusted HR of 3-year mortality for CKD was 1.93 (95 \% CI, 1.55-2.40) compared with those with normal renal function [39]. However, patients with end-stage renal disease (ESRD) receiving hemodialysis had better 3 year survival rate (HR, 0.66; $95 \% \mathrm{CI}$, 0.46-0.94) than those with CKD. This implied that CKD may be associated with poor clearance of circulatory neurotoxic substances that increases the susceptibility to mortality in cirrhotic patients with hepatic encephalopathy. Hung et al. evaluated 6,740 cirrhotic patients who were hospitalized with EVB in 2007 and showed that 
Table 3 Adjusted Risk for 42-Day and One-Year Mortality Stratified by DM, Chronic Kidney Diseases, and Sex

\begin{tabular}{|c|c|c|c|c|c|c|c|c|c|c|c|c|}
\hline & \multicolumn{6}{|c|}{ 42-day mortality } & \multicolumn{6}{|c|}{ One-year mortality } \\
\hline & \multicolumn{3}{|c|}{ Model $1^{a}$} & \multicolumn{3}{|c|}{ Model $2^{b}$} & \multicolumn{3}{|c|}{ Model $3^{a}$} & \multicolumn{3}{|c|}{ Model $4^{b}$} \\
\hline & $\mathrm{HR}$ & $95 \% \mathrm{Cl}$ & $P$ value & $\mathrm{HR}$ & $95 \% \mathrm{Cl}$ & $P$ value & $\mathrm{HR}$ & $95 \% \mathrm{Cl}$ & $P$ value & $\mathrm{HR}$ & $95 \% \mathrm{Cl}$ & $P$ value \\
\hline \multicolumn{13}{|l|}{ All patients } \\
\hline Non-DM \& CKD & Ref. & & & & & & Ref. & & & & & \\
\hline Only DM & 0.72 & $0.50-1.02$ & 0.062 & & & & 0.80 & $0.64-1.02$ & 0.066 & & & \\
\hline Only CKD & 1.25 & $0.62-2.52$ & 0.541 & & & & 1.07 & $0.65-1.76$ & 0.801 & & & \\
\hline $\mathrm{DM}+\mathrm{CKD}$ & 1.99 & $1.03-3.84$ & 0.042 & & & & 1.84 & $1.14-2.98$ & 0.012 & & & \\
\hline$P$ for $D M \times C K D$ interaction & 0.100 & & & & & & & 0.028 & & & & \\
\hline \multicolumn{13}{|l|}{ Women } \\
\hline Non-DM \& CKD & & & & Ref. & & & & & & Ref. & & \\
\hline Only DM & & & & 1.11 & $0.56-2.21$ & 0.772 & & & & 0.77 & $0.49-1.21$ & 0.260 \\
\hline Only CKD & & & & 3.40 & $0.75-15.48$ & 0.113 & & & & 1.30 & $0.39-4.28$ & 0.672 \\
\hline $\mathrm{DM}+\mathrm{CKD}$ & & & & 4.03 & $1.40-11.59$ & 0.010 & & & & 2.84 & $1.31-6.14$ & 0.008 \\
\hline \multicolumn{13}{|l|}{ Men } \\
\hline Non-DM \& CKD & & & & 2.00 & $1.14-3.52$ & 0.016 & & & & 1.61 & $1.13-2.29$ & 0.009 \\
\hline Only DM & & & & 1.25 & $0.67-2.32$ & 0.487 & & & & 1.32 & $0.90-1.92$ & 0.154 \\
\hline Only CKD & & & & 2.02 & $0.83-4.94$ & 0.123 & & & & 1.64 & $0.90-2.98$ & 0.108 \\
\hline $\mathrm{DM}+\mathrm{CKD}$ & & & & 2.93 & $1.14-7.57$ & 0.026 & & & & 2.42 & $1.28-4.57$ & 0.007 \\
\hline$P$ for gender $\times$ diseases interaction & & & & 0.920 & & & & & & 0.559 & & \\
\hline
\end{tabular}

Cl confidence interval, CKD chronic kidney disease, DM diabetes mellitus, HR hazard ratio, Ref reference

${ }^{a}$ Adjusted for sex, age, comorbidities, etiology of cirrhosis, complications of cirrhosis, low income and urbanization

${ }^{\mathrm{b}}$ Adjusted for age, comorbidities, etiology of cirrhosis, complications of cirrhosis, low income and urbanization

ESRD was associated with 1-year mortality (HR,1.50; $95 \% \mathrm{CI}, 1.18-1.91)$, but not a risk factor for 42-day mortality (HR,1.19; $95 \%$ CI, 0.79-1.78) [5]. They implicated that the ESRD-related platelet dysfunction contributed higher EVB and mortality. In our study, we identified that CKD was independent prognostic factor for both 42-day and one-year mortality in cirrhotic patients with first presentation of EVB.

Coexisting DM and CKD are important prognostic factors in cirrhotic patients regardless of the causes of liver diseases. CKD and DM have many long-term complications such as increased immunocompromised status, as well as increased risk of metabolic disorders and cardiovascular events [40-42]. DM increases portal blood flow secondary to fluctuating blood sugar levels leading to an increase in portal pressure $[35,43]$. There is increased bleeding tendency due to uremic platelet dysfunction, use of antiplatelet agents, and anticoagulants [11, 12]. In addition, usage of aspirin increases the occurrence of EVB in cirrhotic patients [13]. These complications could indicate why the 42-day and 1-year overall mortality was higher among the coexisting DM and CKD than DM or CKD individuals. A gender-stratified comparative analysis indicated that coexisting DM and CKD exhibited mortality risk in both genders.
In a cohort study with patients with chronic hepatitis $\mathrm{C}(\mathrm{CHC})$, patients with new-onset diabetes subsequently were found to have an increased risk of developing cirrhosis, or decompensation in those with established cirrhosis [44]. Persico et al. retrospectively evaluated 852 consecutive patients $(726 \mathrm{CHC}$ and 126 chronic hepatitis B) who had undergone liver biopsy [45]. Liver fibrosis (odds ratio [OR], 4.70; 95\%CI, 2.75-8.03) was independent risk factors for the presence of significant steatosis (>30 \%) in patient with CHC. Camma et al. analyzed 104 patients with $\mathrm{CHC}$ cirrhosis (Child-Pugh class $\mathrm{A}$ ) receiving upper gastrointestinal endoscopy [16]. They found a high homeostasis model assessment score (OR, 1.37; $95 \% \mathrm{CI}, 1.01-1.86)$ as an independent predictor of the presence of esophageal varices. Cirrhosis, per se, independently by the viral etiology, may be associated with the development of insulin resistance and diabetes.

Although HCV and HBV infection were not associated with increased risk of mortality, they have been reported to be associated with renal diseases. HCV is also associated with extra-hepatic diseases, including various types of glomerulonephritis, even in the absence of cirrhosis [46]. A high baseline HCV viral load was an independent predictor of CKD [47]. Soma et al. indicated that HCV infection leads to a rapid decline in the renal function of 
patients with diabetic nephropathy [48]. HBV-related renal injury is associated with the deposition of immune complexes of HBV antigens and host antibodies [49]. Untreated chronic HBV infection is also associated with increased risk of CKD [50].

Hepatic steatosis or fatty liver is characterized by lipid accumulation within the cytoplasm of hepatocytes, and includes a spectrum of liver disease from a benign simple steatosis, steatohepatitis, to fibrosis [51]. In Taiwan, the prevalence of non-alcoholic fatty liver disease (NAFLD) is about $11.5 \%$ [52], and the rates are higher in subgroups, from $66.4 \%$ in healthy taxi drivers [53] to $80 \%$ in obese individuals enrolled in weight reduction programs [54]. In a cross-sectional community study that included $11.4 \%$ $(372 / 3,260)$ individuals with elevated alanine aminotransferase (ALT), NAFLD was the most common cause of ALT elevation with a prevalence of $33.6 \%$, and followed by HBV (28.5\%), unexplained cause (21.8 \%), HCV (13.2 \%), both HBV and HCV (2.2\%), and excess alcohol consumption (0.8 \%) [55]. Approximately $1 \%$ of patients with NAFLD develop cirrhosis [56], where as in non-alcoholic steatohepatitis (NASH), the estimated figure is up to $20 \%$ [57]. However, NAFLD was not available in the NHIRD.

Our data included all EVB patients hospitalized in a variety of hospitals in Taiwan; hence, selection bias was minimized. There were some significant limitations to this study. First, the basic laboratory data (e.g. prothrombin times, bilirubin, creatinine, and albumin levels) were not available. For the severity of cirrhosis, Child-Pugh or Model of End-Stage Liver Disease (MELD) scores could not be calculated using the ICD-9 coding in the database. We put previous episodes of decompensation that required hospitalization, such as asictes, hepatic encephalopathy, and SBP into multiple variable analyses. This information could obviate the important limitation of not having laboratory data to calculate the MELD score. Second, the exact cases of NAFLD-related cirrhosis were not available. Data on NAFLD-related cirrhosis are limited and only one case report has been reported in Taiwan [58]. Third, the diagnoses of EVB and other comorbidities were based on ICD-9 codes, and misclassification could be possible. We included cirrhotic patients who were hospitalized with first presentation of EVB and received esophageal variceal ligation or sclerotherapy to minimize ascertainment bias.

\section{Conclusions}

This study provides evidence that coexistence of CKD and DM has a higher impact on 42-day and one-year mortality than DM or CKD individually.

\section{Abbreviations}

ALT: alanine aminotransferase; CHC: chronic hepatitis C; Cl: confidence interval; CKD: chronic kidney disease; COPD: chronic obstructive pulmonary disease; DM: diabetes mellitus; ESRD: end-stage renal disease;
EVB: esophageal variceal bleeding; HBV: hepatitis B virus; HCC: hepatocellular carcinoma; HCV: hepatitis C virus; HR: hazard ratio; ICD-9-CM: International Classification of Diseases, Ninth Revision, Clinical Modification; LHID2005: Longitudinal Health Insurance Database 2005; MELD: Model of End-Stage Liver Disease; NAFLD: non-alcoholic fatty liver disease; NASH: non-alcoholic steatohepatitis; NHIRD: National Health Insurance Research Database; OR: odds ratio; SBP: spontaneous bacterial peritonitis; TB: pulmonary tuberculosis.

\section{Competing interests}

The authors declare that they have no competing interests.

\section{Authors' contributions}

The study was designed by $\mathrm{CCL}_{2} \mathrm{ZHJ}$ and JYH. JYH carried out data analysis, and $C C L, Z H J$ and ONN wrote the paper. All the authors had access to the data. All authors read and approved the final manuscript as submitted.

\section{Acknowledgments}

The authors acknowledge the National Health Research Institute of Taiwan for providing the NHIRD. The descriptions or conclusions herein do not represent the viewpoint of the Bureau.

Received: 4 September 2015 Accepted: 17 February 2016

Published online: 29 February 2016

\section{References}

1. D’Amico G, Garcia-Tsao G, Pagliaro L. Natural history and prognostic indicators of survival in cirrhosis: a systematic review of 118 studies. J Hepatol. 2006;44:217-31.

2. Yang $\mathrm{CH}$, Chiu YC, Chen $\mathrm{CH}$, Chen $\mathrm{CH}$, Tsai MC, Chuah SK, et al. Diabetes mellitus is associated with gastroesophageal variceal bleeding in cirrhotic patients. Kaohsiung J Med Sci. 2014;30:515-20.

3. Augustin S, Muntaner L, Altamirano JT, Gonzalez A, Saperas E, Dot J, et al, Predicting early mortality after acute variceal hemorrhage based on classification and regression tree analysis. Clin Gastroenterol Hepatol. 2009;7:1347-54.

4. Kwon SY, Kim SS, Kwon OS, Kwon KA, Chung MG, Park DK, et al. Prognostic significance of glycaemic control in patients with HBV and HCV-related cirrhosis and diabetes mellitus. Diabet Med. 2005;22:1530-5.

5. Hung TH, Tseng CW, Tseng KC, Hsieh YH, Tsai CC, Tsai CC. Is end stage renal disease a risk factor for the mortality of cirrhotic patients with esophageal variceal bleeding? Hepatogastroenterol. 2014;61:1871-5.

6. del Olmo JA, Pena A, Serra MA, Wassel AH, Benages A, Rodrigo JM. Predictors of morbidity and mortality after the first episode of upper gastrointestinal bleeding in liver cirrhosis. J Hepatol. 2000;32:19-24.

7. Cardenas A, Gines P, Uriz J, Bessa X, Salmeron JM, Mas A, et al. Renal failure after upper gastrointestinal bleeding in cirrhosis: incidence, clinical course, predictive factors, and short-term prognosis. Hepatology. 2001;34:671-6.

8. Pesanti EL. Immunologic defects and vaccination in patients with chronic renal failure. Infect Dis Clin North Am. 2001;15(3):813-32.

9. Kurata M, Tsuboi A, Takeuchi M, Fukuo K, Kazumi T. Association of Metabolic Syndrome with Chronic Kidney Disease in Elderly Japanese Women: Comparison by Estimation of Glomerular Filtration Rate from Creatinine, Cystatin C, and Both. Metab Syndr Relat Disord. 2015; [Epub ahead of print]

10. Holzmann MJ, Carlsson AC, Hammar N, Ivert T, Walldius G, Jungner I, et al. Chronic kidney disease and 10-year risk of cardiovascular death. Eur J Prev Cardiol. 2015; [Epub ahead of print]

11. Escolar G, Diaz-Ricart M, Cases A. Uremic platelet dysfunction: past and present. Curr Hematol Rep. 2005;4:359-67.

12. Kringen MK, Narum S, Lygren I, Seljeflot I, Sandset PM, Troseid AM, et al. Reduced platelet function and role of drugs in acute gastrointestinal bleeding. Basic Clin Pharmacol Toxicol. 2011;108:194-201.

13. De Ledinghen V, Heresbach D, Fourdan O, Bernard P, Liebaert-Bories MP, Nousbaum JB, et al. Anti-inflammatory drugs and variceal bleeding: a case-control study. Gut. 1999:44:270-3.

14. Trombetta M, Spiazzi G, Zoppini G, Muggeo M. Review article: type 2 diabetes and chronic liver disease in the Verona diabetes study. Aliment Pharmacol Ther. 2005;22 Suppl 2:24-7.

15. Francque S, Verrijken A, Mertens I, Hubens G, Van Marck E, Pelckmans P, et al. Visceral adiposity and insulin resistance are independent predictors of the presence of non-cirrhotic NAFLD-related portal hypertension. Int J Obes. 2011;35:270-8. 
16. Camma C, Petta S, Di Marco V, Bronte F, Ciminnisi S, Licata G, et al. Insulin resistance is a risk factor for esophageal varices in hepatitis $C$ virus cirrhosis. Hepatology. 2009;49:195-203.

17. Collins AJ, Foley RN, Chavers B, Gilbertson D, Herzog C, Johansen K, et al. United states renal data system 2011 annual data report: atlas of chronic kidney disease \& end-stage renal disease in the United States. Am J Kidney Dis. 2012;59(1 Suppl 1):A7, e1-420.

18. Cheng JS, Tsai WC, Lin CL, Chen L, Lang HC, Hsieh HM, et al. Trend and factors associated with healthcare use and costs in type 2 diabetes mellitus: a decade experience of a universal health insurance program. Med Care. 2015;53:116-24.

19. Chan TC, Fan I, Liu MS, Su MD, Chiang PH. Addressing health disparities in chronic kidney disease. Int J Environ Res Public Health. 2014;11:12848-65.

20. Kuo RN, Lai MS. Comparison of Rx-defined morbidity groups and diagnosisbased risk adjusters for predicting healthcare costs in Taiwan. BMC Health Serv Res. 2010;10:126.

21. Liao YH, Lin CC, Li TC, Lin JG. Utilization pattern of traditional Chinese medicine for liver cancer patients in Taiwan. BMC Complement Altern Med. 2012;12:146

22. Liu ME, Tsai SJ, Chang WC, Hsu CH, Lu T, Hung KS, et al. Population-based 5-year follow-up study in Taiwan of dementia and risk of stroke. PLoS One. 2013;8:e61771

23. Lee YT, Nfor ON, Tantoh DM, Huang JY, Ku WY, Hsu SY, et al. Herpes zoster as a predictor of HIV infection in Taiwan: a population-based study. PLoS One. 2015;10:e0142254

24. National Health Insurance Research Database, Taiwan. http://nhird.nhri.org. tw/en/Data_Subsets.html. Accessed 31 Oct 2015.

25. Perz JF, Armstrong GL, Farrington LA, Hutin YJ, Bell BP. The contributions of hepatitis $B$ virus and hepatitis $C$ virus infections to cirrhosis and primary liver cancer worldwide. J Hepatol. 2006;45(4):529-38.

26. Chen $\mathrm{CH}$, Yang PM, Huang GT, Lee HS, Sung JL, Sheu JC. Estimation of seroprevalence of hepatitis B virus and hepatitis $C$ virus in Taiwan from a large-scale survey of free hepatitis screening participants. J Formos Med Assoc. 2007:106:148-55.

27. Tsai JF, Chang WY, Jeng JE, Ho MS, Lin ZY, Tsai JH. Hepatitis B and C virus infection as risk factors for liver cirrhosis and cirrhotic hepatocellular carcinoma: a case-control study. Liver. 1994;14:98-102.

28. Kao JH. Hepatitis B, vaccination and prevention of hepatocellular carcinoma. Best Pract Res Clin Gastroenterol. 2015;29:907-17.

29. Mayor S. Liver cancer in Taiwan falls after universal hepatitis B vaccination. BMJ. 1997;315:7.

30. Fortune B, Garcia-Tsao G. Current management strategies for acute esophageal variceal hemorrhage. Curr Hepatol Rep. 2014;13:35-42.

31. Chang TT, Liaw YF, Wu SS, Schiff E, Han KH, Lai CL, et al. Long-term entecavir therapy results in the reversal of fibrosis/cirrhosis and continued histological improvement in patients with chronic hepatitis B. Hepatology. 2010:52:886-3.

32. Carbonell N, Pauwels A, Serfaty L, Fourdan O, Levy VG, Poupon R. Improved survival after variceal bleeding in patients with cirrhosis over the past two decades. Hepatology. 2004;40:652-9.

33. Garcia-Compean D, Jaquez-Quintana JO, Maldonado-Garza H. Hepatogenous diabetes. Current views of an ancient problem. Ann Hepatol. 2009;8:13-20.

34. Majid S, Azam Z, Shah HA, Salih M, Hamid S, Abid S, et al. Factors determining the clinical outcome of acute variceal bleed in cirrhotic patients. Indian J Gastroenterol. 2009;28:93-5.

35. Pugliese D, Lee SS, Koshy A, Cerini R, Ozier Y, Lebrec D. Systemic and splanchnic hemodynamic effects of intravenous hypertonic glucose in patients with cirrhosis. Hepatology. 1988;8:643-6.

36. Jeon HK, Kim MY, Baik SK, Park HJ, Choi H, Park SY, et al. Hepatogenous diabetes in cirrhosis is related to portal pressure and variceal hemorrhage. Dig Dis Sci. 2013;58:3335-41.

37. Hung TH, Tsai CC, Hsieh YH, Tsai CC, Tseng CW, Tsai JJ. Effect of renal impairment on mortality of patients with cirrhosis and spontaneous bacterial peritonitis. Clin Gastroenterol Hepatol. 2012;10:677-81.

38. Verkade MA, van Druningen CJ, Vaessen LM, Hesselink DA, Weimar W, Betjes MG. Functional impairment of monocyte-derived dendritic cells in patients with severe chronic kidney disease. Nephrol Dial Transplant. 2007:22:128-38

39. Hung TH, Tseng CW, Tseng KC, Hsieh YH, Tsai CC, Tsai CC. Effect of renal function impairment on the mortality of cirrhotic patients with hepatic encephalopathy: a population-based 3-year follow-up study. Medicine. 2014;93:e79.

40. Saito I. Epidemiological evidence of type 2 diabetes mellitus, metabolic syndrome, and cardiovascular disease in Japan. Circ J. 2012;76:1066-73.

41. Koh GC, Peacock SJ, van der Poll T, Wiersinga WJ. The impact of diabetes on the pathogenesis of sepsis. Eur J Clin Microbiol Infect Dis. 2012;31:379-88.

42. Kato S, Chmielewski M, Honda H, Pecoits-Filho R, Matsuo S, Yuzawa Y, et al. Aspects of immune dysfunction in end-stage renal disease. Clin J Am Soc Nephrol. 2008:3:1526-33.

43. Moreau R, Chagneau C, Heller J, Chevenne D, Langlet $P$, Deltenre $P$, et al. Hemodynamic, metabolic and hormonal responses to oral glibenclamide in patients with cirrhosis receiving glucose. Scand J Gastroenterol. 2001;36:303-8.

44. Huang YW, Yang SS, Fu SC, Wang TC, Hsu CK, Chen DS, et al. Increased risk of cirrhosis and its decompensation in chronic hepatitis $C$ patients with new-onset diabetes: a nationwide cohort study. Hepatology. 2014:60:807-14

45. Persico M, Masarone M, La Mura V, Persico E, Moschella F, Svelto M, et al. Clinical expression of insulin resistance in hepatitis $C$ and $B$ virus-related chronic hepatitis: differences and similarities. World J Gastroenterol. 2009;15:462-6.

46. Arase Y, Ikeda K, Murashima N, Chayama K, Tsubota A, Koida I, et al. Glomerulonephritis in autopsy cases with hepatitis $C$ virus infection. Intern Med. 1998:37:836-40.

47. Satapathy SK, Lingisetty CS, Williams S. Higher prevalence of chronic kidney disease and shorter renal survival in patients with chronic hepatitis $C$ virus infection. Hepatol Int. 2012;6:369-78.

48. Soma J, Saito T, Taguma Y, Chiba S, Sato H, Sugimura K, et al. High prevalence and adverse effect of hepatitis C virus infection in type II diabetic-related nephropathy. J Am Soc Nephrol. 2000;11:690-9.

49. Bhimma R, Coovadia HM. Hepatitis B virus-associated nephropathy. Am J Nephrol. 2004;24:198-211.

50. Chen YC, Su YC, Li CY, Hung SK. 13-year nationwide cohort study of chronic kidney disease risk among treatment-naive patients with chronic hepatitis $B$ in Taiwan. BMC Nephrol. 2015:16:110.

51. Serfaty L, Lemoine M. Definition and natural history of metabolic steatosis: clinical aspects of NAFLD, NASH and cirrhosis. Diabetes Metab. 2008;34:634-7.

52. Chen $\mathrm{CH}$, Huang $\mathrm{MH}$, Yang JC, Nien CK, Yang CC, Yeh YH, et al. Prevalence and risk factors of nonalcoholic fatty liver disease in an adult population of taiwan: metabolic significance of nonalcoholic fatty liver disease in nonobese adults. J Clin Gastroenterol. 2006:40:745-52.

53. Tung $\mathrm{TH}$, Chang $\mathrm{TH}$, Chiu WH, Lin TH, Shih HC, Chang MH, et al. Clinical correlation of nonalcoholic fatty liver disease in a Chinese taxi drivers population in Taiwan: Experience at a teaching hospital. BMC Res Notes. 2011:4:315.

54. Hsiao TJ, Chen JC, Wang JD. Insulin resistance and ferritin as major determinants of nonalcoholic fatty liver disease in apparently healthy obese patients. Int J Obes Relat Metab Disord. 2004;28:167-72.

55. Chen $\mathrm{CH}$, Huang MH, Yang JC, Nien CK, Yang CC, Yeh YH, et al. Prevalence and etiology of elevated serum alanine aminotransferase level in an adult population in Taiwan. J Gastroenterol Hepatol. 2007;22:1482-9.

56. Dam-Larsen $S$, Franzmann M, Andersen IB, Christoffersen $P$, Jensen LB, Sorensen $\mathrm{Tl}$, et al. Long term prognosis of fatty liver: risk of chronic liver disease and death. Gut. 2004:53:750-5.

57. McCullough AJ. The clinical features, diagnosis and natural history of nonalcoholic fatty liver disease. Clin Liver Dis. 2004;8:521-33.

58. Tang CP, Huang YS, Tsay SH, Chang FY, Lee SD. Nonalcoholic fatty liver disease manifesting esophageal variceal bleeding. J Chin Med Assoc. 2006;69:175-8. 